\title{
Myasthenia gravis: a pathologic approach
}

\author{
J.S.J.M. Hounjet \\ Maastricht University \\ j.hounjet@student.maastrichtuniversity.nl
}

\section{Abstract}

Our immune system protects us from all kinds of pathogens our body faces every day. However, the immune system can also initiate an immune response against our own proteins. This is called autoimmunity. Myasthenia gravis (MG) is a rare autoimmune disease in which patients show severe muscle weakness, due to antibody production against proteins of the neuromuscular junction, which is the connection between nerve and muscle. At this moment, three different forms of myasthenia gravis are identified. Muscle-specific tyrosine kinase (MUSK)-MG is a form of MG in which patients produce antibodies, predominantly immunoglobulin (Ig)G4, against MuSK. This paper is a summary of a B cell immortalization project, in which antibodies produced by MuSK-MG patients are investigated. The aim of this study was to sequence and generate antibodies produced by these patients in vitro to learn more about the pathological mechanism of MUSK-MG. Blood-derived B cells of MuSK-MG patients were immortalized by the Epstein Bar Virus and stimulated to grow and produce antibodies in culture. Genes of these antibodies were amplified and analyzed. These amplification products were cloned in vectors and will be transfected in a human embryonic kidney (HEK)-cell line. This study showed that the $B$ cell immortalization method worked and that MUSK-MG patients predominantly produce IgM antibodies against MuSK. This is very striking as literature states that these patients predominantly produce IgG4 against MuSK. It is suggested that this high production of $\operatorname{IgM}$ is due to the absence of the heavy chain isotype switch from IgM to IgG. Moreover, as the pathologic mechanism of MuSK-MG is still unknown, IgM may play an important pathological role.

\section{Keywords}

Myasthenia gravis, muscle specific tyrosine kinase, autoantibodies, B cell immortalization, immunoglobulin M 


\section{Introduction}

The immune system protects the human body from all kinds of pathogens. The importance of our immune system for health is dramatically illustrated by the frequent observation that individuals with defective immune responses are susceptible to serious, often lifethreatening infections. Conversely, abnormal immune responses can cause autoimmune diseases, with serious morbidity and mortality (1). Autoimmune diseases are a range of diseases, in which the immune response to self-antigens results in damage or dysfunction of tissues. Autoimmune diseases can be systemic or can affect specific organs or body systems (2). Autoantibodies are mainly produced by B cells. When naïve B cells (CD22+ cells) are activated by antigen, they proliferate and differentiate into effector cells, also called plasma cells. These effector cells produce and secrete antibodies $(1,3)$. These antibodies, also called immunoglobulins exist of two heavy and two light chains. There are different subtypes of heavy chains, like: IgG, IgM and IgA. The light chain belong either to the kappa or lambda type (1). The human antibody response exists of two phases. The first response is the primary antibody response, in which predominantly immunoglobulin $M(\operatorname{Ig} M)$ is produced. In the secondary antibody response there is an antibody switch to the IgG isotype, which is called heavy chain isotype switching.

Myasthenia gravis (MG) is a rare autoimmune disease characterized by the production of autoantibodies against proteins of the postsynaptic membrane in the neuromuscular junction (NMJ). This leads to weakness and fatigability of skeletal and extra-ocular muscles (4). Three subclasses of myasthenia gravis are identified. Patients with the "MuSK-MG" form are of importance in this study. These patients process antibodies against the muscle-specific tyrosine kinase (MUSK) (4-6). MuSK is a postsynaptic neuromuscular protein involved in acetylcholine receptor (AChR) clustering (7-9), which is important for the communication between nerve and muscle. These antibodies belong predominantly to the $\operatorname{lgG}_{4}$ subclass and are not associated with damage to the neuromuscular junction (4). However, also IgG1 can be produced by these patients (10). The main effect of MuSK antibodies at the neuromuscular junction is still unclear. MuSK antibodies may not only inhibit MUSK function directly, but also increase the turnover of MuSK, thereby further reducing its activity (9). These findings suggest that MuSK-MG may be different in etiological and pathological mechanisms, compared to AChR-MG, in which patients produce autoantibodies against the AChR. In MuSK-MG, the postsynaptic machinery becomes structurally and functionally disorganized by a significant reduction in AChR clustering $(7,9)$. MuSK-MG is an early-onset myasthenia gravis (EOMG) that occurs predominantly in middle-aged woman, and is often severe $(7,8,10)$. The risk of myasthenic crisis is particularly high, and the chances of achieving complete stable 
remission are significantly lower than in AChR-MG (10).Current treatments are steroid and immunosuppressive drugs for patients with MuSK-MG (5).

This paper is about a project on MUSK-MG with a pathologic approach, in which MuSK-MG patients' antibodies are investigated. The aim of this study was to sequence and generate antibodies of MuSK-MG patients in vitro to gain more insight into the pathological mechanisms of this form of myasthenia gravis and to test new drugs without the use of patients or patients' blood in future. The hypothesis is that the immortalized B cell clones will produce IgG4 or IgG1 antibodies against MuSK. To achieve this, B cells were isolated from MuSK-MG patients' blood, cultured, and immortalized with the Epstein Barr virus (EBV). After immortalization, B cell clones were stimulated to grow and produce antibodies. The supernatants of the $B$ cell clones were screened for antibody production against MuSK. From positive B cell clones RNA was extracted, converted to DNA, and inserted in an immunoglobulin vector, which will be transfected in a HEK-cell line. With this method, MUSK-MG patient antibody producing cells will be obtained.

\section{Material and methods}

\section{Patients}

The exact characteristics of the five patients, from which blood was taken, are unknown. The only characteristic known was that the patients were classified as MuSK-MG patients and that they were from Leiden and Maastricht University. In general, MuSK-MG has an early onset of disease, occurs predominantly in middle-aged woman and is often severe $(7,8,10)$.

\section{Isolation of human B cells}

The cell fraction of peripheral mononuclear blood cells (PMBCs), including B cells, was isolated from whole MuSK-MG patient blood by Density Gradient Centrifugation using Ficoll (Histopaque ${ }^{\circledR}$ 10771, Sigma). A schematic overview of the procedures is illustrated (figure 1). To isolate CD22+MuSK+lgG+ and CD22+MuSK+lgG- B cells, isolated PMBCs were stained with fluorescently labelled CD22+ and IgG antibodies and MuSK (recombinant protein, extracellular domain, $60 \mathrm{kD}, 1 \mathrm{mg} / \mathrm{ml}$ ) fluorescently labelled. By use of fluorescence activated cell sorting (FACS) using a FACSAria II cell sorter (BD Biosciences) the two subsets of $B$ cells were isolated. After isolation, $B$ cells were immortalized as described previously by Vrolix et al (11). In short, selected B cells were seeded in $96 \mathrm{U}$-bottom micro well plates (treated for cell culture, Costar) in advanced RPMI 1640 medium (Roswell Park Memorial Institute, Gibco) supplemented with 1\% L-glutamine, HEPES, 10\% fetal bovine serum, 
$1 \mathrm{mM}$ sodium pyruvate, $50 \mathrm{U} / \mathrm{ml}$ penicillin, and $50 \mathrm{U} / \mathrm{ml}$ streptomycin. The isolated cells were immortalized during 2 weeks in the presence of $1 \times 105$ irradiated (30 Gy) peripheral mononuclear blood cells of healthy donors used as feeder cells needed by the B cells to grow, Eptein-Barr virus (EBV)-containing supernatant $(3.4 \times 108$ copies $/ \mathrm{ml})$ and $1 \mu \mathrm{g} / \mathrm{ml}$ CpG2006 (TLR-9 agonist, Invivogen), which stimulates the cells to grow and produce antibodies. To make a better distinction between immortalized $B$ cells and feeder cells, $1 \times 105$ irradiated (50 Gy) Wi38 cells (ATCC ${ }^{\circledR}$ CCL-75TM human lung fibroblasts, 3 months gestation fetus, ATCC) were also used as feeder cells, as they have a different shape compared to the immortalized B cell clones. After two weeks, cells were restimulated with $1 \mu \mathrm{g} / \mathrm{ml} \mathrm{CpG} 2006$ and $50 \mathrm{U} / \mathrm{ml}$ interleukin-2 (IL-2, Roche) to grow and produce antibodies for two weeks, after which the immortalization was verified by microscopic examination of cell growth and screening of culture supernatants for antibody production. Growthpositive and antibody producing clones were kept in culture until they grew confluently in a $75 \mathrm{~cm} 2$ flask and could be frozen in liquid nitrogen at $-80^{\circ} \mathrm{C}$.

\section{Antibody production screening}

Culture supernatants of $\mathrm{CD} 22+\mathrm{MuSK}+\operatorname{lgG}+$ and $\mathrm{CD} 22+\mathrm{MuSK}+\operatorname{lgG}$ - immortalized B cell clones were screened for human IgG production by use of enzyme-linked sorbent assay (ELISA). With this method a plate with plastic wells was coated with $50 \mu \mathrm{l} /$ well of an antihuman IgG antibody (unconjugated, Jackson ImmunoResearch), diluted 1:200 in coating buffer (50 mM sodium carbonate, pH 9.6). After incubation, the plates were blocked with $4 \%$ non-fat dry milk (Blotting Grade Blocker, Biorad) in PBS (salt solution). After incubation, blocking buffer was removed and $30 \mu$ l of incubation buffer (1\% BSA, 0.02\% Tween-20 in PBS, $\mathrm{pH}$ 7.4) and $15 \mu \mathrm{l}$ of supernatant of B cell clones was added to the wells. Human IgG (HUMAN IgG purified Immunoglobulin, $5.6 \mathrm{mg} / \mathrm{ml}$, Sigma) ranging from 1000 to 0.975 $\mathrm{ng} / \mathrm{ml}$, was included as a positive control, and culture medium was used as a negative control. After incubation, six washing steps were performed with washing buffer (0.05\% Tween-20 in PBS). After washing, $50 \mu \mathrm{l} /$ well of antihuman IgG conjugated with peroxidase (PO) (Jackson ImmunoResearch), diluted 1:20,000, was added to all wells. After incubation, washing was performed and $100 \mu \mathrm{l} /$ well of substrate solution ( $1 \%$ citrate buffer, $0.1 \%$ TMB, $0.0001 \% \mathrm{H}_{2} \mathrm{O}_{2}$ in distillated water) was added. If the culture supernatant contained any human IgG antibodies the peroxidase would convert the substrate solution, which leads to a stable blue colour. The reaction was stopped by adding $50 \mu \mathrm{l}$ of $2 \mathrm{M} \mathrm{H}_{2} \mathrm{SO}_{4}$. After stopping the reaction, absorption was measured at $450 \mathrm{~nm}$ for 1.0 seconds with an ELISA reader (Perkin Elmer 2030).

The same ELISA was used to screen supernatants for specific antibodies. The culture 
supernatants of growing, immortalized B cell clones, both IgG positive and negative, were screened for IgG against MUSK. In these ELISAs MUSK (1:1,000, extracellular domain, 60 kD, $1 \mathrm{mg} / \mathrm{ml}$ stock concentration) was used to coat the plates and sera of a MuSK-MG patient, diluted 1:500, was used as a positive control, instead of a standard curve, to see if the B cell clones produced IgG against MuSK. Supernatants of growing, immortalized B cell clones were also screened for IgM production against MUSK for four subsequent weeks. The ELISA was performed according to the same protocol as the IgG against MUSK ELISA, except for the secondary antibody and positive control. Supernatants of both IgG negative and positive sorted, growing B cell clones were screened. As a secondary antibody, peroxidaseconjugated anti-human IgM (Jackson ImmunoResearch), diluted 1:2000, was used. After immortalization of the B cell clones, growing and antibody producing B cell clones were screened each week, to see if they were still positive for IgG or IgM against MuSK.

\section{RNA extraction and immunoglobulin gene amplification}

From MuSK antibody, either IgG or IgM, producing B cell clones, RNA was extracted to obtain the immunoglobulin (antibody) genes. After RNA extraction, first-strand cDNA synthesis was performed. In this first-strand CDNA synthesis a small single stranded DNA (primer) binds to the single stranded RNA. In presence of a reverse transcriptase, which is an enzyme that can elongate from the primer on, the primer was elongated. After elongation an RNAse was added, which leads to the degradation of the RNA strand. The result of this procedure was a single stranded cDNA. After first-strand CDNA synthesis, CDNA was stored at $-80^{\circ} \mathrm{C}$ or directly used for amplification by polymerase chain reaction (PCR). Different primers were used to amplify the immunoglobulin DNA fragments. In this PCRs a difference was made in heavy and light chain gene amplifications. After the amplification by PCR, obtained immunoglobulin genes were analyzed by agarose (UltrapureTM Agarose, Invitrogen) gel electrophoresis and visualized by ethidium bromide staining. In this gel elektrophoresis, gene fragments run in the gel dependent of their length in base paires. A 100 Base-Pair (bp) ladder (Invitrogen) was used, as the heavy chain products were around $400 \mathrm{bp}$ (12) and the light chain products were around $300 \mathrm{bp}$ (13) in length. When the immunoglobulin genes were visualized in the gels, amplification products were purified to obtain a pure solution of amplified immunoglobulin genes. 


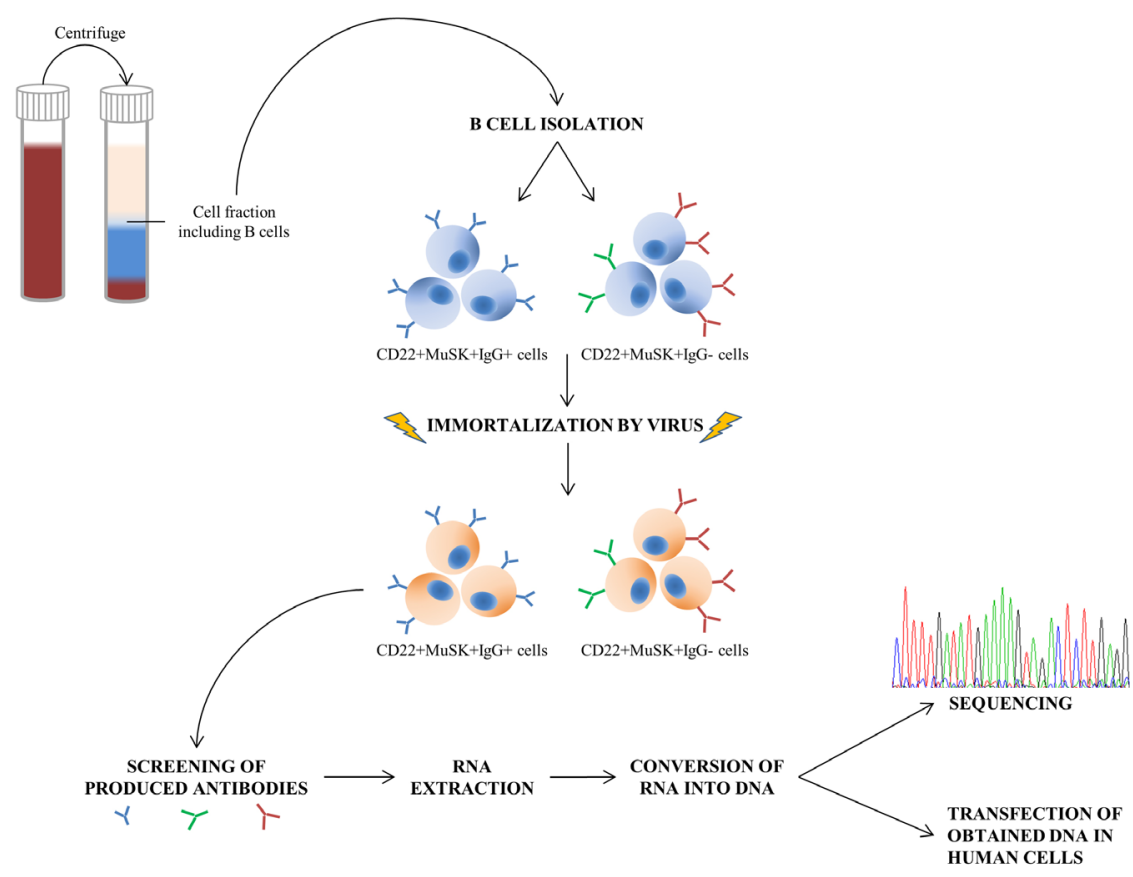

Figure 1. Schematic overview of B cell isolation, immortalization, stimulation and screening. Blood-derived PBMCs (cell fraction including B cells) of MuSK-MG patients were isolated. From PBMCs, B cells were isolated by FACS. Two groups of cells were cultured: $C D 22+M u S K+l g G+$ and CD22+MuSK+lgG- sorted B cells. After one week, B cells were immortalized by the Epstein-Barr virus and stimulated with $\mathrm{CpG}_{2006}$. After two weeks, $\mathrm{B}$ cells were restimulated with $\mathrm{CpG}_{2006}$ and IL-2 for two weeks. After stimulation, immortalization was verified by microscopic examination of cell growth and screening the culture supernatants for antibody production. RNA was extracted from antibody producing B cell clones and converted to DNA by PCR. The obtained immunoglobulin genes were sequenced and will be transfected in human cells.

\section{Sequencing}

Sequencing is a method to obtain the genetic code of DNA. To obtain the sequence of the purified immunoglobulin genes of the immortalized B cell clones, the Sanger sequencing method was used, which is based on the selective incorporation of chain-terminationg dideoxynucleotides, which are the building blocks of DNA.The sequence reactions were purified by use of MicrospinTM G50 Columns (GE Healthcare). The purified sequencing reactions were added to a $0.1 \mathrm{ml}$ reaction plate (MicroAMP ${ }^{\circledR}$ Optical 96-well, Applied Biosystems). Sequences were analysed by the genetic analyser $\mathrm{ABI} 300$ (Applied Biosystems) and Chromas Lite (Version 2.1.1). 


\section{Transfection of immunoglobulin genes in human embryonic kidney (HEK)-cell line}

To transfect the immunoglobulin genes obtained from MUSK-MG patients blood, these genes had first to be placed in vectors. A vector is a circular double stranded DNA that can ligate a DNA fragment and has a antibiotic resistance to make sure that only bacteria that toke up the vector will grow on petridishes that contain this specific antibiotic. Four different vectors were used. Two heavy chain vectors: IgG1 and IgG4 and two light chain vectors: lambda and kappa. These vectors were first amplified by bacteria ( $\mathrm{DH} 10 \mathrm{~B}$ competent cells; E. coli) which is called transformation. After amplification of the vector, vector DNA was extracted by lysis of the bacteria. This vector DNA was measured and stored at $4^{\circ} \mathrm{C}$. Both obtained vector DNA and immunoglobulin genes were cut by restriction enzymes (New England Biolabs). These restrictions were analyzed by agarose gel electrophoresis and visualized by ethidium bromide staining. As the cut, and thereby linearized fragments ran slower through the gel than the uncut, circular vector, the gels were used to see if the restrictions were complete. These restriction enzymes cut DNA in specific sites, which made it possible to place the immunoglobulin genes in the vector DNA in the right position. This binding of genes and vector and thereby re-circularization is called ligation. The ligation products, the vectors including the immunoglobulin genes, were amplified in bacteria (DH10a competent cells; E. coli, Invitrogen).

The ligation products will be extracted from the bacteria and these products will be placed, also called transfected, in a human embryonic kidney (HEK) cell-line. With this method antibodies produced by MUSK-MG patients will be produced by these cells in vitro.

\section{Results}

\section{Immortalized B cell clones produce IgG and IgM against MuSK}

Antibody production screening resulted in immortalized $B$ cell clones with activity against MuSK. From the first patient 3 out of $25 \mathrm{CD} 22+M u S K+\lg G+$ sorted B cell clones produced IgG against MuSK and 8 out of 57 CD22+MuSK+lgG- sorted and growing cell clones produced IgM against MuSK. From the second patient 1 out of $3 \lg G+$ sorted $B$ cell clones produced IgG against MuSK and 23 out of 54 IgG- sorted B cell produced IgM against MuSK. From the third patient the 2 IgG+ sorted B cell clones were both negative for IgG against MuSK. Moreover, 4 out of 18 IgG- sorted B cell clones of the third patient produced IgM against MuSK. From the fourth patient only one of the 9 IgG- sorted growing B cell clone produced IgM against MuSK. None of the $9 \mathrm{IgG}+$ sorted and growing B cell clones produced IgG against MuSK. From the fifth patient all $17 \mathrm{lgG}+$ sorted and growing B cell clones were negative for IgG against MuSK. However, 30 out of 78 IgG-sorted and growing 
B cell clones were IgM against MuSK positive. Moreover, the subsequent supernatants, which were screened every week after stimulation, showed striking differences in antibody production.

\section{Ten immunoglobulin genes were extracted for sequencing and transfection}

From 10 immortalized B cell clones, which were repeatedly positive in the ELISA screening, RNA was extracted (table 1). From the first patient RNA was extracted from $2 \mathrm{~B}$ cell clones. From the $\mathrm{G}_{41} \mathrm{~B}$ cell clone $\operatorname{Ig} \mathrm{G} \lambda$ genes were extracted. From the $\mathrm{F}_{42} \mathrm{~B}$ cell clone $\lg \mathrm{M} \mathrm{k}$ genes were extracted. From the second patient $\operatorname{Ig} G \lambda$ genes were extracted from $B$ cell clone F62. From the third patient no immunoglobulin genes were extracted as these clones did not show growing and high antibody production in the screening. From the fourth patient $\lg M \mathrm{k}$ genes were extracted from B cell clone $C 61$. From the fifth patient $6 \lg M$ heavy chain genes were extracted. From five of these clones $\lambda$ genes were extracted. From one of these clones both $\mathrm{\kappa}$ and $\lambda$ genes were extracted.

Table 1. Extracted immunoglobulin genes from immortalized B cell clones of five MuSK-MG patients. From all patients one or more immunoglobulin genes were extracted, except for the third patient. B cell clones of this patient did not show high antibody production in the screening.

\begin{tabular}{cccc}
\hline Patient & B cell clone & Heavy chain & Light chain \\
\hline 1 & G41 & $\lg$ & $\lambda$ \\
& F42 & $\lg M$ & $\mathrm{~K}$ \\
\hline 2 & F62 & $\lg$ & $\lambda$ \\
\hline 3 & $*$ & $*$ & $*$ \\
\hline 4 & C61 & $\operatorname{lgM}$ & $\mathrm{k}$ \\
\hline 5 & F52 & $\lg M$ & $\lambda$ \\
& F74 & $\operatorname{lgM}$ & $\lambda$ \\
& B115 & $\lg M$ & $\lambda$ \\
& B55 & $\lg M$ & $\lambda$ \\
& E45 & $\lg M$ & $\lambda$ \\
& E103 & $\lg M$ & $\kappa \lambda$
\end{tabular}

\section{Sequencing of immunoglobulin genes showed incomplete sequences}

Sequencing of DNA extracted from immortalized B cell clones showed the four nucleotides of DNA in four different colours. This sequencing showed incomplete sequences. The peaks of the nucleotides were low and peaks of different nucleotides had the same height, which made it hard to analyze the genetic code. Some sequencing reactions went well 
(figure 2). However, the sequences were not complete. The first and last part, around 100 base pairs (bp), of the sequences were missing.

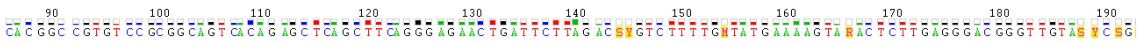

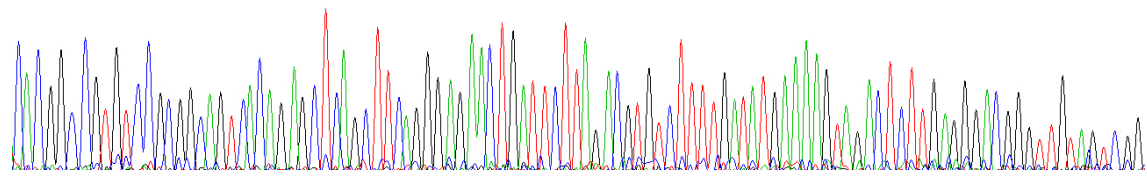

Figure 2. Example of sequencing result. This sequence was obtained from an IgM heavy chain amplification of a B cell clone ( $\left.F_{52}\right)$ of the fifth patient. The nucleotide peaks were low. Moreover, the sequence was incomplete. Blue: $\mathrm{C}$, green: A, red: T, black: $\mathrm{G}$.

\section{Vector amplification obtained high concentrations of pure DNA}

All four vectors were amplified in bacteria. All transformations showed many growing bacteria, although there were differences in the amount of colonies, which are the bacteria that grew on the plates, between the vectors. For the IgG1 vector a high amount of transformed bacteria grew on the plates. A high DNA concentration (mean: $520 \mathrm{ng} /$ $\mu l)$ was extracted from four colonies. Moreover, the DNA was pure and no contamination occurred. The plates of the IgG4 transformed bacteria were far less crowded than the IgG1 plates. However, the obtained DNA concentrations were high (mean: $382 \mathrm{ng} / \mu \mathrm{l}$ ) and contained pure, uncontaminated vector DNA. On the plates of the kappa vector grew far less colonies than on the IgG1 plates, but the obtained DNA concentrations were also high (mean: $458 \mathrm{ng} / \mu \mathrm{l}$ ), pure and uncontaminated. The plates of the lambda vector were crowded, like the plates of IgG1. The obtained DNA concentrations were high (mean: 545 $\mathrm{ng} / \mu \mathrm{l})$ and pure.

\section{Restrictions of the vectors and obtained immunoglobulin genes were complete}

All four vectors were digested by two restriction enzymes. The IgG1 and IgG4 vectors were digested by the restriction enzymes EcoRI and Nhel (figure 3). The control reaction 
(C), without enzyme, showed two bands for the $\operatorname{lgG}_{1}$ and $\operatorname{lgG}_{4}$ vector, which showed the uncut, circular formations of the vector. However, the intensity of the bands of the $\operatorname{lgG}_{4}$ control reaction was very low. The EcoRI digestion was absent in both IgG1 and IgG4 vectors, as two bands appeared at the same level as the control reaction without enzyme (C), which showed that this restriction enzyme was not digesting the vector. However, after increasing reaction volume and incubation time and decreasing the amount of DNA, the digestions by EcoRI were complete. The Nhel digestion was almost complete for the IgG1 vector and complete for the IgG4 vector, as it showed one band, which appeared higher in the gel than the lower band of the control, which indicate that the vector linearized (dashed square), and thereby cut.

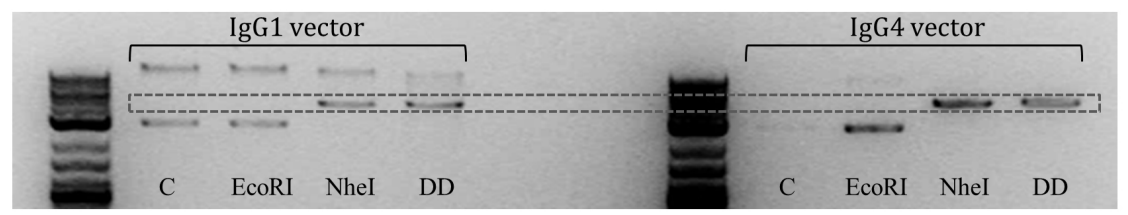

Figure 3. Digestion of IgG1 and IgG4 vector by EcoRI and Nhel. The left part of the gel contains the IgG1 vector restriction. The level of the linearized (cut) vector is shown by the dashed square. The right part shows the $\operatorname{lgG}_{4}$ vector restriction. Both $\lg G_{1}$ and $\lg _{4}$ vectors showed no digestion for EcoRI. Nhel showed almost complete digestion for IgG1 and complete digestion for $\operatorname{lgG}_{4}$ vector. C: control reaction without enzyme, EcoRI: reaction with only EcoRI present, Nhel: reaction with only Nhel present. DD: double digestion, both EcoRI and Nhel were present.

The double digestion reactions showed both almost complete digestion. EcoRI and BsiWI cut the kappa vector and EcoRI and Avrll digested the lambda vector. Both vectors showed complete restriction after digestion by the restriction enzymes. As all restriction were complete, the obtained immunoglobulin gene fragments were also restricted by the same enzymes as their vectors. These restriction products will be ligated, which means that the obtained immunoglobulin genes will be placed in the digested vectors. The ligation products will be amplified in bacteria. Once amplified the ligation products will be obtained by bacteria lysis and will be transfected in human embryonic kidney (HEK)cells to generate the antibodies produced by MuSK-MG patients in vitro.

\section{Discussion/Conclusion}

This pathologic study, in which antibodies produced by MuSK-MG patients were investigated, showed that the immortalized B cell clones produced IgG and IgM 
antibodies with reactivity against MUSK. From these antibodies the majority belonged to the IgM isotype. Moreover, this screening showed that the sorting by FACS went well, as all CD22+MuSK+lgG- sorted B cells were negative for IgG. Furthermore, the subsequent supernatants, which were screened every week after stimulation, showed striking differences in antibody production. This may be due to the fact that taken off the supernatants may have damaged the cells, which leads to a decreased antibody production in the following screening week.

From ten B cell clones that produced antibodies against MuSK, RNA was extracted and the immunoglobulin genes, both heavy and light chains, were amplified. This resulted in 2 $\operatorname{lgG} \lambda$ genes, $2 \lg M \kappa$ genes and $6 \lg M \lambda$ genes, from which one showed also amplifications for the kappa light chain. The gene amplifications confirmed that the majority of the produced antibodies against MUSK belonged to the IgM isotype, with a predominance of lambda light chains. It was expected that the produced antibodies by the immortalized $\mathrm{B}$ cell clones would predominantly belong to the $\operatorname{lgG}_{4}$ isotype as described in literature $(5,9,14)$. However, the majority of the immortalized B cell clones showed to produce IgM antibodies against MuSK. Moreover, there was a predominance of lambda light chains, which is striking as normally $60-75 \%$ of the light chains belong to the kappa type $(15,16)$. Sequencing of the obtained immunoglobulin genes showed incomplete sequences. According to literature, human heavy chains are around $400 \mathrm{bp}$ in length (12) and light chains are around $300 \mathrm{bp}$ in length (13). The obtained sequences of the heavy chains were missing around $100 \mathrm{bp}$. Furthermore, nucleotide peaks were low and often peaks of different nucleotides had the same height, which made it hard to obtain the correct sequence. At the moment, we are trying to improve our sequencing results. Moreover, ligation products will be sequenced and will be compared to the first, incomplete sequences.

Vector cloning went well, as all four vectors were cloned. However, the selection and growing of the vectors showed some differences in efficiency. This may be due to differences in vectors or bacteria used for transformation. From all four vectors, a high amount of pure, uncontaminated DNA was obtained. As a high amount of $\operatorname{lgM}$ amplifications were obtained, also an IgM vector will be used for cloning. The subsequent restrictions of the vectors showed incomplete restriction by EcoRl. However, after increasing the reaction volume and incubation time and decreasing the amount of DNA, the digestions by EcoRI were complete. As the restrictions of the vectors were complete, PCR products were restricted as well. These digested PCR products were ligated with the appropriate restricted vectors. The ligation products will be amplified in bacteria and the amplified ligation products will be transfected in a HEK cell line to generate antibodies produced by MuSK-MG patients in vitro. 
The limitations of this study were the low number of patients and also the small amount of blood used for the immortalization. This is only a small part of the whole mechanism, which makes it hard to make an overall conclusion on MuSK-MG. However, this study showed a predominance of IgM production against MuSK. This could mean that IgM plays an important role in the pathology of MUSK-MG, as was already suggested by Yamamoto et al in 1991 (17) and Hofstad et al in 1992 (18). Both purposed that the heavy chain isotype switch from IgM to IgG was missing in seronegative patients and thereby these patients produce IgM for an indefinite time. However, MuSK-MG was not identified yet (2001) during these studies (10). In 2003, IgM antibodies were again found in seronegative patients, but not in the MuSK-MG fraction (19). Normally, these IgM autoantibodies are not found during established disease (20). However, this study confirms that IgM may play an important role in the pathology of MuSK-MG. Moreover, it is confirmed that IgM is an very efficient activator of immune system, which indicates it importance in autoimmunity (18).

The pathophysiology of IgM is rarely a focus in human autoimmune disease (20). Therefore, more research is needed to explore the pathophysiological role of IgM to delineate the exact role of IgM in autoimmunity and MuSK-MG. IgM may be an important player or missing link in MuSK-MG. Moreover, if we succeed to produce the obtained antibodies in vitro, these cells can be used to investigate MuSK-MG to learn more about the pathological mechanisms. Furthermore, the antibody producing cells can be used to test the effects of common and new drugs in vitro, without the need of MuSK-MG patients' blood. More research is recommended to unravel the role of IgM in the pathology of MuSK-MG.

\section{Role of the student}

Judith Hounjet was an undergraduate student working under the supervision of Dr Gisela Nogales-Gadea and Dr Mario Losen when the research in this report was performed. The topic was proposed by the supervisor. The research from the antibody screening until the restrictions of the obtained antibody genes and vectors were performed by the student. Moreover, the student expanded her internship to go on with the project and also writing for publication were done by the student.

\section{Acknowledgments}

I would like to thank Dr Mario Losen for making this interesting and educational internship possible for me. Moreover, I want to thank Dr Gisela Nogales Gadea for her daily support and confidence in my work. Furthermore, I want to thank the Psychiatry and Neuropsychology department of Maastricht University for their support and answers to all my questions. 


\section{References}

1. Abbas A, Lichtman A. Basic Immunology: Functions and Disorders of the Immune System. Updated zrd ed. Philadelphia Saunders Elsevier; 2011.

2. Ngo S, Steyn F, McCombe P. Gender differences in autoimmune disease. Frontiers in neuroendocrinology. 2014.

3. Alberts B, Johnson A. Molecular Biology of the Cell. 4th ed. New York: Garland Science 2002.

4. Avidan N, Le Panse R, Berrih-Aknin S, Miller A. Genetic basis of myasthenia gravis-a comprehensive review. J Autoimmun. 2013.

5. Motomura M. Pathogenic antibodies in myasthenia gravis. Brain and nerve. 2010;62(4):411-8.

6. Zisimopoulou P, Evangelakou P, Tzartos J, Lazaridis K, Zouvelou V, Mantegazza R, et al. A comprehensive analysis of the epidemiology and clinical characteristics of anti-LRP4 in myasthenia gravis. J Autoimmun. 2013.

7. Huijbers MG, Lipka A, Plomp J, Niks E, Maarel S, Verschuuren J. Pathogenic immune mechanisms at the neuromuscular synapse: the role of specific antibody-binding epitopes in myasthenia gravis. Journal of internal medicine. 2014;275(1):12-26.

8. Lavrnic D, Losen M, Vujic A, De Baets M, Hajdukovic L, Stojanovic V, et al. The features of myasthenia gravis with autoantibodies to MuSK. Journal of Neurology, Neurosurgery \& Psychiatry. 2005;76(8):1099-102.

9. Gomez AM, Van Den Broeck J, Vrolix K, Janssen SP, Lemmens MA, Van Der Esch E, et al. Antibody effector mechanisms in myasthenia gravis-pathogenesis at the neuromuscular junction. Autoimmunity. 2010;43(56):353-70.

10. Sieb JP. Myasthenia gravis: an update for the clinician. Clinical \& Experimental Immunology. 2014;175(3):408-18.

11. Vrolix K, Fraussen J, Losen M, Stevens J, Lazaridis K, Molenaar PC, et al. Clonal heterogeneity of thymic B cells from early-onset myasthenia gravis patients with antibodies against the acetylcholine receptor.J Autoimmun. 2014 .

12. Williams DG, Taylor PC. Clonal analysis of immunoglobulin mRNA in rheumatoid arthritis synovium: characterization of expanded IgG3 populations. European journal of immunology. 1997;27(2):476-85.

13. Kawasaki K, Minoshima S, Schooler K, Kudoh J, Asakawa S, de Jong PJ, et al. The organization of the human immunoglobulin lambda gene locus. Genome Research. 1995;5(2):125-35.

14. Querol L, Illa I. Myasthenia gravis and the neuromuscular junction. Current opinion in neurology. 2013;26(5):45965.

15. Heilmann C, Barington T. Distribution of kappa and lambda light chain isotypes among human blood immunoglobulin-secreting cells after vaccination with pneumococcal polysaccharides. Scand J Immunol 1989;29(2):159-64.

16. Molé CM, Béné MC, Montagne PM, Seilles E, Faure GC. Light chains of immunoglobulins in human secretions. Clinica chimica acta. 1994;224(2):191-7.

17. Yamamoto T, Vincent A, Ciulla TA, Lang B, Johnston I, Newsom-Davis J. Seronegative myasthenia gravis: A plasma factor inhibiting agonist-induced acetylcholine receptor function copurifies with IgM. Annals of neurology. 1991;30(4):550-7.

18. Hofstad H, Ulvestad E, Gilhus N, Matre R, Aarli J. Myasthenia gravis muscle antibodies examined by ELISA: IgG and IgM antibodies characterize different patient subgroups. Acta neurologica scandinavica. 1992;85(4):233-8.

19. Vincent A, Bowen J, Newsom-Davis J, McConville J. Seronegative generalised myasthenia gravis: clinical features, antibodies, and their targets. The Lancet Neurology. 2003;2(2):99-106.

20. Duarte-Rey C, Bogdanos DP, Leung PS, Anaya J-M, Gershwin ME. IgM predominance in autoimmune disease: genetics and gender. Autoimmun Rev. 2012;11(6):A404-A12. 